\title{
Study of antimicrobial effect of some plants of Lamiaceae family on Escherichia coli species isolated from children with urinary tract infection
}

\author{
Hossein Emad Momtaz ${ }^{1}$, Shirin Moradkhani ${ }^{2,3^{*}}{ }^{\circledR}$, Mohammad Yousef Alikhani ${ }^{4}$, Farzaneh \\ Esnaashari ${ }^{5}$, Mojdeh Afkhami ${ }^{1}$ \\ ${ }^{1}$ Division of Pediatric Nephrology, Besat Hospital, Hamadan University of Medical Sciences, Hamadan, Iran \\ ${ }^{2}$ Medicinal Plants and Natural Products Research Center, Hamadan University of Medical Sciences, Hamadan, Iran \\ ${ }^{3}$ Department of Pharmacognosy and Pharmaceutical Biotechnology, School of Pharmacy, Hamadan University of Medical Sciences, \\ Hamadan, Iran \\ ${ }^{4}$ Department of Microbiology, School of Medicine, Hamadan University of Medical Sciences, Hamadan, Iran \\ ${ }^{5}$ Department of Community Medicine, School of Medicine, Hamadan University of Medical Sciences, Hamadan, Iran
}

\section{A R T I C L E I N F O}

Article Type:
Original

\section{Article History:}

Received: 20 October 2017

Accepted: 24 January 2018

Published online: 26 February 2018

\section{Keywords:}

Lamiaceae family

Escherichia coli

$\mathrm{MBC}$

MIC

Children

Urinary tract infection

\begin{abstract}
A B S T R A C T
Introduction: Urinary tract infection (UTI) has a high prevalence in children. It is an important cause of renal scar, chronic renal disease, failure to thrive and hypertension. The unnecessary consumption of antibiotics results in the bacterial resistance to most common drugs. In addition, possible drug allergic reaction and side effects should be considered. Therefore, finding new antimicrobial compounds is necessary.

Objectives: In this study, the antimicrobial effect of some plants of Lamiaceae family on Escherichia coli in comparison with common antibiotics used in UTI was investigated.

Materials and Methods: In this experimental study, the hydroalcoholic extract of 13 plant species from Lamiaceae family were prepared and their antimicrobial effects were assessed by the agar well diffusion method. In addition, minimum inhibitory concentration (MIC) and minimum bactericidal concentration $(\mathrm{MBC})$ were investigated using the serial dilution method. The zone of inhibition (ZOI) of the growth of E. coli was compared between the studied extracts and five most common antibiotics in the treatment of UTI.

Results: The mean diameter of ZOI was significantly higher for amikacin than the studied extracts and other antibiotics. Among the herbal extracts, the highest ZOI was observed for Nepeta crispa. In addition, the MIC and MBC of N. crispa were significantly lower than other extracts.

Conclusion: According to the side effects and relatively high cost of chemical and synthetic drugs, use of herbal extracts, such as $N$. crispa may be recommended as an alternative treatment of UTI with $E$. coli. The results of present study are promising to introduce new candidates in the treatment of UTI.
\end{abstract}

Implication for health policy/practice/research/medical education:

Considering high prevalence of urinary tract infection (UTI) in children and its important complications, finding new natural treatments with fewer side effects and lower microbial resistance than usual antibiotics may be valuable. In this study authors tried to show the antimicrobial effect of some herbal extracts against $E$. coli for possible use of those as complementary treatment of UTI.

Please cite this paper as: Momtaz HE, Moradkhani Sh, Alikhani MY, Esnaashari F, Afkhami M. Study of antimicrobial effect of some plants of Lamiaceae family on Escherichia coli species isolated from children with urinary tract infection. J Renal Inj Prev. 2019;8(1):38-43. Doi: 10.15171/jrip.2019.09.

\section{Introduction}

Urinary tract infection (UTI) is a common disease in children. About $1 \%-3 \%$ of girls and $1 \%$ of boys suffer from UTI. Gram-negative bacteria in intestine are the main cause of UTI. Escherichia coli is the most common bacteria in UTI, causing $75 \%-90 \%$ of all infections, followed by Klebsiella and Proteus (1). The majority of UTI cases are ascending infections. The bacteria originated from stool flora are colonized in perineal area and reach the bladder through urethra. The management of UTI 
patients is faced with microbial resistance. Antibiotics currently used in the treatment of UTI gradually lose their effects. Although some antibiotics, such as cotrimoxazole, ampicillin, and amoxicillin are inexpensive, the administration of them is not suitable in many countries because of their ineffectiveness (2). Risk factors, such as genitourinary system anomalies, vesicoureteral reflux, history of antibiotic administration, and recurrent UTI are important factors in increasing the incidence of antibiotic resistance. Unnecessary consumption of antibiotics results in the bacterial resistance to available and commonly used drugs, which in turn leads to increase the administration of newer and more expensive drugs. Continuing this trend results in bacterial resistance to newer drugs.

Medicinal plants and herbal medicine have been one of the most important issues in medical and pharmacological sciences. The use of medicinal plants and natural products has a long history. Following numerous failures in the use of chemical drugs and the advent of new resistant microorganisms, human beings have been producing various medicinal products with herbal origin. Lamiaceae family has more than 2500 members and mostly grows in temperate regions. They are indigenous to the Mediterranean region. Eighty-one species of Lamiaceae family are used for medicinal purposes in Iran. They have been used in traditional treatment of bloating and indigestion, insomnia, psychological disorders such as depression, anxiety and for memory improvement (3). The main compounds in the Lamiaceae family plants are flavonoids and polyphenols. These compounds are inhibitors of cholinesterase and their antioxidant and antiinflammatory effects have been reported in several studies (4-6). In the previous studies, the antimicrobial effects of the essential oils of some members of Lamiaceae family, such as thyme, savory, marjoram, basil and lavender have been investigated (7-9).

To the best of our knowledge, there is no published study investigating the antibacterial effects of medicinal plants on E. coli, the most common pathogen of UTI. Therefore, the present study was performed to investigate the antibacterial effects of 13 species of Lamiaceae family, including Salvia spinosa, Salvia multicaulis, Salvia virgata, Stachys lavandulifolia, Stachys inflata, Nepeta crispa, Nepeta fissa, Phlomis olivieri, Phlomis aucheri, Phlomis herba-venti, Phlomis anisodonta, Thymus daenensis, and Teucrium polium against E. coli, and compared with antibacterial effects of the common antibiotics used in UTI, including cefixime, nitrofurantoin, co-trimoxazole, amikacin, and ceftriaxone.

\section{Materials and Methods}

The plant species were collected from the populations growing wild in Hamadan province, west of Iran. A voucher number was deposited to each specimen in herbarium of Department of Pharmacognosy, School of Pharmacy, Hamadan University of Medical Sciences,
Hamadan, Iran. The hydroalcoholic extracts of 13 plant species from Lamiaceae family were prepared and their antimicrobial effects against $E$. coli were investigated. The air-dried plant materials were powdered and macerated in hydroalcohol (85\%) for three times, each time three days. The filtrates were dried by means of rotary evaporator. The obtained extracts were kept in a refrigerator until use.

Bacteria isolated from urine samples of children with UTI admitted in the pediatric ward of Besat hospital were studied. After identification, the sensitivity and resistance of the bacteria to the studied extracts and five commonly used antibiotics in the treatment of UTI, including cefixime, nitrofurantoin, co-trimoxazole, amikacin, and ceftriaxone were assessed.

\section{Agar well diffusion assay}

To assess the antibacterial activities of the studied extracts, the agar well diffusion method was used. The Mueller-Hinton agar (MHA, Merck Co, Germania) was used according to a previously published method (10) and results were interpreted according to the Clinical \& Laboratory Standards Institute (CLSI) guidelines (11). The bacteria were dissolved in Phosphate buffer and their turbidity was compared with $0.5 \mathrm{McFarland}$ standards $\left(10^{8}\right.$ microorganisms per $\left.\mathrm{mL}\right)$. Agar surface of each plate was streaked with a sterile cotton swab with the bacterial strains. Agar plate was punched with a sterile cork borer of $4 \mathrm{~mm}$ size and $50 \mu \mathrm{L}$ of the 8 different dilutions (from $0.4 \mathrm{mg} / \mathrm{mL}$ to $13.5 \mathrm{mg} / \mathrm{mL}$ ) of the extract was poured with a micropipette in the bore. In each culture plate, one well was considered as a blank. The plates were allowed to standby for 30 minutes. The plates were incubated at $37^{\circ} \mathrm{C}$ for 24 hours.

\section{Determination of MIC and $M B C$}

To determine minimum inhibitory concentration (MIC) of each extract, 10 tubes containing different dilutions of the extracts as well as positive and negative controls were used. Different dilutions of each extract from $0.4 \mathrm{mg} / \mathrm{mL}$ (tube 1) to $13.5 \mathrm{mg} / \mathrm{mL}$ (tube 8 ) were prepared in nutrient broth culture media (Merck Co., Germany) with one $\mathrm{mL}$ of bacteria suspension. The negative control contained 9 $\mathrm{mL}$ of the culture medium and one $\mathrm{mL}$ of the bacterial suspension. The positive control contained $9 \mathrm{~mL}$ of the culture medium with $1 \mathrm{~mL}$ of each extract. All tubes were incubated at $37^{\circ} \mathrm{C}$ for 24 hours and then assessed for their turbidity caused by the growth of inoculated bacteria. For determination of minimum bactericidal concentration (MBC), a sample was taken from all tubes where the bacterium was inhibited in the MIC assay. After the cultivation of MHA and 24 hours incubation, the results were studied.

\section{Ethical issues}

The study was conducted in accordance with the principles of Declaration of Helsinki 1996 version and its later 
amendments and also Good Clinical Practice standards. The study protocol and other study related documents were reviewed and approved by research council of Hamadan University of Medical Sciences (\# 9211153891).

\section{Statistical analysis}

Data were analyzed using SPSS version 23.0. For descriptive statistics mean \pm SD was used. After checking for variables normality because of abnormal distribution, KruskalWallis $\mathrm{H}$ test was used. For comparison of groups, post hoc test (Bonferroni) was used and $P<0.5$ was considered as significant.

\section{Results}

\section{Comparison of the diameter of zone of inhibition}

Figure 1 shows the zone of inhibition (ZOI) for the studied extracts and antibiotics. Among antibiotics amikacin had the largest mean diameter of ZOI and cotrimoxazole had the lowest. The difference of ZOI of amikacin was significant with all antibiotics except nitrofurantoin and ceftriaxone. Zone of inhibition of cotrimoxazole was significantly smaller than all other antibiotics.

Nepeta crispa had the largest mean diameter of ZOI among herbal extracts and S. spinosa had the smallest ZOI. This difference of ZOI of N. crispa was significant only with $S$. spinosa and $S$. multicaulis.

The mean diameter of ZOI for amikacin was larger than those of the studied extracts and other antibiotics. There was a significant difference with ZOI of all antibiotics and extracts except of ZOI of N. crispa, P. olivieri, T. polium, nitrofurantoin and ceftriaxone.

ZOI for nitrofurantoin was statistically different from the extracts of $S$. spinosa, S. multicaulis and cotrimoxazole $(P<0.05)$. In addition, the ZOI for $N$. crispa and $P$. olivieri were significantly larger than those of cotrimoxazole $(P<0.05)$. Interestingly $\mathrm{ZOI}$ of cefixime was not significantly different from that of all herbal extracts $(P>0.05)$. Co-trimoxazole showed the smallest ZOI among all studied materials and it was significantly different from all studied antibiotic and extracts, except for S. inflata, S. multicaulis and S. spinosa $(P<0.05)$.

\section{MIC and $M B C$}

Figures 2 and 3 show MBC and MIC of studied herbal extracts, respectively. The MIC for $N$. crispa was significantly lower than other extracts $(P<0.05)$. The MIC for $P$. olivieri was significantly lower than MIC of S. spinosa, $P$. aucheri, $P$. herba-venti, $T$. daenensis and $T$. polium $(P<0.05)$. The highest MIC was observed for the extracts of T. polium, which was not significantly different from all other extracts except $N$. crispa and P. olivieri. The lowest values of MBC were observed for N. crispa which was significantly lower than those of other extracts $(P<0.05)$ except $P$. olivieri. T. polium extract had highest $\mathrm{MBC}$ but was not significantly different from other extracts except $N$. crispa and P. olivieri. After N. crispa and P. olivieri, S. multicaulis had rather low MBC, but that was

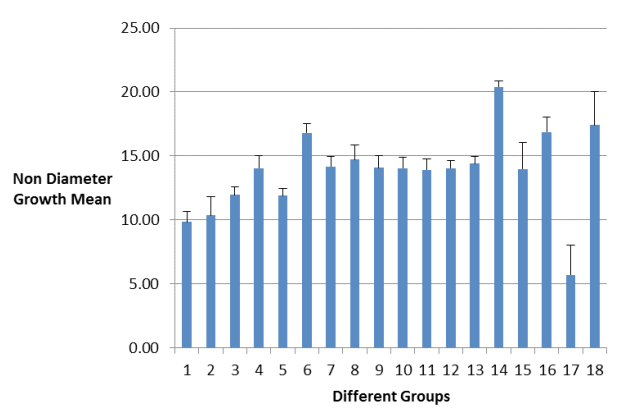

Figure 1. Comparison of mean zone of inhibition (ZOI) between different herbal extracts and antibiotics.

1. Salvia spinosa; 2. Salvia sulticaulis; 3. Salvia virgata; 4. Stachys lavandulifolia; 5 . Stachys inflata; 6 . Nepeta crispa; 7. Nepeta fissa; 8. Phlomis olivieri; 9. Phlomis aucheri; 10. Phlomis herba-venti; 11. Phlomis anisodonta; 12. Thymus daenensis; 13. Teucrium polium. 14. Amikacin; 15. Cefixime; 16, Nitrofurantoin; 17, Cotrimoxazole; 18 , Ceftriaxone.

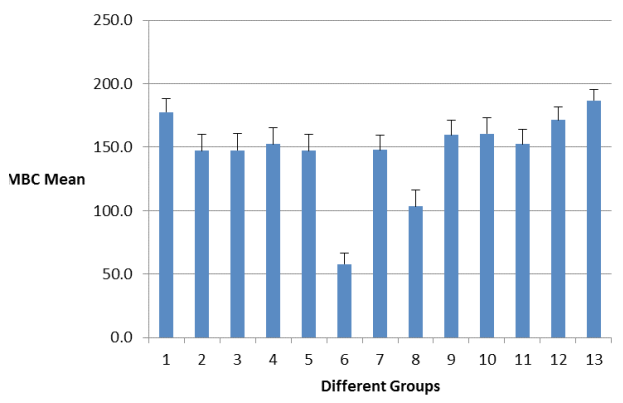

Figure 2. Comparison of minimum bactericidal concentration (MBC) between different herbal extracts and antibiotics.

1. Salvia spinosa; 2. Salvia sulticaulis; 3. Salvia virgata; 4. Stachys lavandulifolia; 5. Stachys inflata; 6 . Nepeta crispa; 7. Nepeta fissa; 8. Phlomis olivieri; 9. Phlomis aucheri; 10. Phlomis herba-venti; 11. Phlomis anisodonta; 12. Thymus daenensis; 13. Teucrium polium.

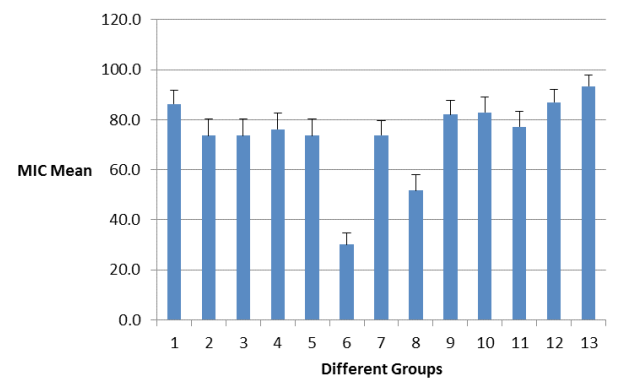

Figure 3. Comparison of minimum inhibitory concentration (MIC) between different herbal extracts and antibiotics.

1. Salvia spinosa; 2. Salvia sulticaulis; 3. Salvia virgata; 4. Stachys lavandulifolia; 5. Stachys inflata; 6. Nepeta crispa; 7. Nepeta fissa; 8. Phlomis olivieri; 9. Phlomis aucheri; 10. Phlomis herba-venti; 11. Phlomis anisodonta; 12. Thymus daenensis; 13. Teucrium polium.

not significantly different with other extracts About $23.8 \%$ and $9.52 \%$ of the isolated bacteria were resistant to $S$. multicaulis and S. spinosa extracts, respectively. None of the isolated $E$. coli were resistant 
to other studied extracts. Among isolated E. coli, $80.95 \%, 33.33 \%, 28.57 \%$, and $9.52 \%$ were resistant to cotrimoxazole, ceftriaxone, cefixime, and nitrofurantoin, respectively. None of the isolated bacteria were resistant to amikacin.

\section{Discussion}

UTI is a common disease in children which is mainly caused by intestinal bacteria (E. coli) (1). The early detection and prevention of the disease is important in children and results in the prevention of kidney damages in them (12). Antibiotics that have commonly been used in the treatment of UTI, despite of low cost are not suitable in many countries because of ineffectiveness (13). The unnecessary consumption of antibiotics results in the bacterial resistance to available and commonly used drugs. Therefore, given the advantages of herbal products over chemical and synthetic drugs, such as lower cost and fewer side effects, better antimicrobial activity, and more popularity in society, the use of them will be of a great importance in solving this problem.

Because of their therapeutic effects, Lamiaceae family has been used from ancient time to cure different diseases. Several studies showed the presence of polyphenols, flavonoids, and terpenoids in the extracts of different members of this family (14). The antimicrobial, antifungal, antivirus, anti-inflammation, and anticancer effects of some members of Lamiaceae family have been reported (15-17). In this study, the antibacterial effects of 13 species of Lamiaceae family were compared with commonly used antibiotics in UTI. The most effective antibiotic was amikacin. No bacterial resistance was observed for this antibiotic and its ZOI diameter was significantly larger than other studied antibiotics. In accordance with our findings, Tarhani et al reported that E. coli resistance to amikacin was $2.4 \%$ and the mean resistance of $3.1 \%$ was reported for all studied microorganisms (2). Co-trimoxazole acts by inhibiting bacterial synthesis of dihydrofolic acid and blocking production of tetrahydrofolic acid, so blocks two consecutive steps in the biosythesis of nucleic acids and proteins essential to bacteria. In the present study, co-trimoxazole was found to have the least antibacterial effect so that its ZOI diameter was significantly smaller than other studied antibiotics and most of herbal extracts. The bacterial resistance to this antibiotic was $80.95 \%$. In the study of Tarhani et al, the highest bacterial resistance was found for ampicillin (88.25\%) and amoxicillin (86.6\%), followed by co-trimoxazole (75.6\%).The bacterial resistance to this antibiotic in the cultivated $E$. coli samples was $54.3 \%$ (2), which is in accordance with our findings. In this study, the bacterial resistance to cefixime, nitrofurantoin, and ceftriaxone were $28.57 \%, 9.52 \%$, and $33.33 \%$, respectively and their ZOI diameters were 14.0 $\mathrm{mm}, 16.86 \mathrm{~mm}$, and $17.41 \mathrm{~mm}$, respectively. Cefixime and ceftriaxone belong to the third cephalosporin antibiotics which inhibit the mucopeptide generation and prevent the formation of cell wall leading to osmotic instability and death of the bacteria. Nitrofurantoin is a macrolide, which seems that inhibits $50 \mathrm{~S}$ ribosomal subunit and thus results in inhibition of protein synthesis and bacteria death. In the study of Tarhani et al, the bacterial resistances to cefixime, nitrofurantoin, and ceftriaxone were $4.7 \%, 11.8 \%$, and $3.1 \%$, respectively (2). The bacterial resistance to cefixime and ceftriaxone in the present study was higher than the study by Tarhani et al. This finding can be explained, at least in part, by the recent consumption of these antibiotics in most patients whose urine samples were collected. In the study of Prado et al, the highest bacterial resistance was observed for co-trimoxazole (52\%) and ampicillin, respectively (18). In the study of Sahm et al, $92.8 \%$ and $7.7 \%$ of $E$. coli isolations were resistant to co-trimoxazole and nitrofurantoin, respectively (19). Furthermore, Ryu et al reported that the most suitable antibiotic for the treatment of UTI caused by gram-negative bacteria was amikacin (20). Similar to our findings, In the study by Amiri et al, the bacterial resistance to co-trimoxazole, cefixime, nitrofurantoin, ceftriaxone, and amikacin in E. coli isolations from the urine samples of subjects with UTI were $80.4 \%, 97.9 \%, 9.50 \%, 95.9 \%$, and $17.5 \%$, respectively (21).

In the present study, the antimicrobial effects of 13 species of Lamiaceae family were investigated. Our findings showed that $N$. crispa had the highest antibacterial effects. Its ZOI diameters was $16.82 \mathrm{~mm}$ which was significantly lower than that of amikacin. However, it was not significantly different from the ZOI diameters for ceftriaxone and nitrofurantoin. The ZOI diameters for $N$. crispa and $P$. olivieri extracts were significantly larger than that of co-trimoxazole.

The MIC and MBC of N. crispa extract were significantly lower than other studied extracts. In addition, no resistance of $E$. coli to these extracts was observed.

Nepeta crispa is a member of Lamiaceae family, which has been used as sedative, anti-flatulence, antispasm, anticough, antiasthma, aseptic agent, and diuretic (22). The effective ingredients in these plants are 1,8-cineole (62.8\%), 4a $\alpha-7 \alpha-7 \mathrm{a} \alpha$-nepetalactone $(10.3 \%)$ and $4 \mathrm{a} \beta-7 \alpha-$ $7 \mathrm{a} \beta$-nepetalactone (9.2\%) (23). In accordance with our observations, Sonboli et al found that the ZOI of E. coli was $16 \mathrm{~mm}$ for $N$. crispa and its inhibitory effects were observed even at 1:16 dilution. Sonboli et al also found that gram-positive bacteria, such as Staphylococcus aureus and Bacillus subtilis were the most sensitive species to the essential oil of N. crispa (24). Likewise, Mahboubi et al reported that the inhibitory and bactericidal effects of Nepeta crispa on E. coli were higher than those of vancomycin, amphotricine B, and gentamicin (25). Phlomis represents 70 species in Iran, of which 8 species are exclusive to Iran. Different species of Phlomis have been used in the treatment of diabetes, stomach ulcer, hemorrhoids and inflammations (26). In addition, the antibacterial, anticancer, and antioxidant activities have been reported for them $(27,28)$. The main compounds of the essential oil of $P$. olivieri, obtained from its leaves, were 
normal-octane $(28.77 \%)$, camphor (13.32\%), 1,8-cineol (12.24\%), a-pinene (7.8\%), and germacrene D (6.57\%) (29). In accordance with our findings, Turker et al, in a study of anticancer and antibacterial effects of some species of Phlomis showed that $P$. olivieri had significant antibacterial effects against E. coli, S. aureus, Klebsiella pneumonia, Pseudomonas aeruginosa, and S. aeruginosa (30).

In our study, the extracts of N. crispa, P. olivieri, T. daenensis, S. lavandulifolia, $P$. herba-venti, had relatively high antibacterial effects and showed higher ZOI in comparison with cefixime although there was no significant difference. However, their ZOI was significantly larger than that of co-trimoxazole.

Salvia virgata, S. inflata, S. multicaulis, P. anisodonta and S. spinosa showed weak antimicrobial effects and their ZOI was smaller than that of cefixime. However, the difference was not significant for any of these extracts.

Although it seems that overall inhibitory effects of herbal extracts may be lower than antibiotics, our findings showed that the proportion of resistant isolates to herbal extracts $(2.57 \%)$ were significantly lower than those resistant to antibiotics (30.47\%). Therefore, with increasing the dose of herbal extracts, it is possible to have remarkable inhibitory effects (similar to antibiotics) against bacteria with fewer side effects than chemical and synthetic antibiotics. Bazzaz et al found that the extracts of S. spinosa, S. virgata, and T. polium had no effects on the growth of E. coli (15). Mojab et al showed that the extract of T. daenensis had inhibitory effects against grampositive bacteria such as S. aureus, Micrococcus luteus, Enterococcus faecalis, and Streptococcus pyogenes, but they did not observe any inhibitory effects against gramnegative bacteria, including E. coli (31).

Since the plants of Lamiaceae family contain flavonoids, it is noted that most of the antibacterial activity of them is because of the formation of complexes between flavonoids and extracellular proteins, soluble proteins, and cell wall proteins. In addition, hydrophobic flavonoids have the ability to destruct the bacteria plasma membrane $(32,33)$. Therefore, antibacterial effects of these plants may be attributed to their different amounts of flavonoids. Thus, the isolation and purification of effective antibacterial compounds in these plants have a great importance in the treatment of UTIs.

\section{Conclusion}

Because of the high bacterial resistance to co-trimoxazole, its use in the early treatment of UTIs in our population is not recommended. On the other hand, amikacin and nitrofurantoin showed higher effectiveness than other studied antibiotics and thus they are recommended to be used as suitable antibiotics in the early treatment of UTIs. Considering the important fact that nitrofurantoin has no significant parenchymal concentration in kidney and is not recommended for treatment of pyelonephritis. The studied herbal extracts showed acceptable antibacterial effects. Therefore, because of the high cost and side effects of chemical and synthesized antibiotics, the use of herbal extracts, especially $N$. crispa may be recommended as an alternative treatment of UTI.

\section{Limitations of our study}

One of limitations of our study was that we were unable to exactly specify strains of $E$. coli isolated from our patients and study their sensitivity and resistance to antibiotics and herbal extracts. Another limitation was that we only determined MIC and MBC of herbal extracts and not antibiotics due to some microbiologic technical problems.

\section{Acknowledgements}

The authors thank the Vice Chancellor of Research and Technology of Hamadan University of Medical Sciences for funding and supports.

\section{Authors' contribution}

HEM conceived the study. MYA performed the experiments. MA collected samples. FE analyzed the data. HEM and ShM drafted the final manuscript. All authors read, revised and approved the final manuscript.

\section{Conflicts of interest}

The authors declare no potential conflicts of interest.

\section{Ethical considerations}

Ethical issues (including plagiarism, data fabrication, double publication) have been completely observed by the authors.

\section{Funding/Support}

The study was approved by research council of Hamadan University of Medical Sciences (\# 9211153891).

\section{References}

1. Kliegman R, Behrman RE, Nelson WE. Nelson Textbook of Pediatrics. 20 ed. Vol. 2. Phialdelphia, PA: Elsevier; 2016.

2. Tarhani F, Kazemi A H. Evaluation of antibiotic resistance in patients with urinary tract infection, Khorramabad Madani hospital 2001-2002. Yafte. 2004;5:39-463.

3. Balali P, Soodi M, Saeidnia S. Protective effects of some medicinal plants from Lamiaceae family against betaamyloid induced toxicity in PC12 cell. Tehran Univ Med J. 2012;1:70.

4. Adams M, Gmünder F, Hamburger M. Plants traditionally used in age related brain disorders-A survey of ethnobotanical literature. J Ethnopharmacol. 2007; 113:363-81. doi: 10.1016/j.jep.2007.07.016

5. Topcu G, Kusman T. Lamiaceae family plants as a potential anticholinesterase source in the treatment of Alzheimer's disease. Bez Sci 2014;1:1-25. doi: 10.14235/bs.2014.233

6. Vladimir-Knežević S, Blažeković B, Kindl M, Vladić J, Lower-Nedza AD, Brantner AH. Cetylcholinesterase inhibitory, antioxidant and phytochemical properties of selected medicinal plants of the Lamiaceae family. Molecules. 
2014;19:767-82. doi: 10.3390/molecules19010767.

7. Radaelli M, da Silva BP, Weidlich L, Hoehne L, Flach A, da Costa LA, et al. Antimicrobial activities of six essential oils commonly used as condiments in Brazil against Clostridium perfringens. Braz J Microbiol. 2016;47:424-30. doi: 10.1016/j.bjm.2015.10.001.

8. Sakkas H, Papadopoulou C. Antimicrobial activity of basil, oregano, and thyme essential oils. J Microbiol Biotechnol. 2017;27:429-438. doi: 10.4014/jmb.1608.08024.

9. Rezvanpanah S, Rezaei K, Golmakani M-T, Razavi SH. Antibacterial properties and chemical characterization of the essential oils from summer savory extracted by microwaveassisted hydrodistillation. Braz J Microbiol. 2011;42:14531462. doi: 10.1590/S1517-838220110004000031.

10. Abbas SZ, Hussain K, Hussain Z, Ali R, Abbas T. Antibacterial activity of different soaps available in local market of Rawalpindi (Pakistan) against daily encountered bacteria. Pharm Anal Acta. 2016;7:522. doi: 10.4172/21532435.1000522 .

11. Clinical and Laboratory Standards Institute. Performance standards for antimicrobial susceptibility testing. 26th ed. Wayne, PA: CLSL; 2016

12. SedighiI I, Solgi A, Alikhani MY, Momtaz HE, Mihani F. Comparison of two different disk diffusion agar tests in determination of antibiotic susceptibility for E. coli isolated from urinary tract infection in pediatrics. Sci J Hamadan Univ Med Sci. 2010;17:17-20. [Persian].

13. Wright SW, Wrenn KD, Haynes MLR. Trimethoprimsulfamethoxazole resistance among urinary coliform isolates. J Gen Intern Med. 1999;14:606-609. doi: 10.1046/j.1525-1497.1999.10128.x.

14. Bonjar GH. Antibacterial screening of plants used in Iranian folkloric medicine. Fitoterapia. 2004;75:231-5. doi: 10.1016/j.fitote.2003.12.013.

15. Bazzaz BS, Haririzadeh G. Screening of Iranian plants for antimicrobial activity. Pharm Biol. 2003;41:573-83. doi: $10.1080 / 13880200390501488$.

16. Sarac N, Ugur A. The in vitro antimicrobial activities of the essential oils of some Lamiaceae species from Turkey. J Med Food. 2009;12:902-7. doi: 10.1089/jmf.2008.0089.

17. Chalabian F, Norouzi Arasi H, Moosavi S. A study of growth inhibitory effect of essential oils of seven species from different families on some kinds of microbes. J Med Plants. 2003;3:37-42.

18. Prado V, Trucco O, Duran C, Mamani R, Royer M. Profile of antimicrobial resistance of agents causing urinary tract infections in Chilean children. PRONARES surveillance program. Rev Med Chil. 2001;129:877-85.

19. Sahm DF, Thornsberry C, Mayfield DC, Jones ME, Karlowsky JA. Multidrug-resistant urinary tract isolates of Escherichia coli: Prevalence and patient demographics in the United States in 2000. Antimicrob Agents Chemother. 2001;45:1402-1406. doi: 10.1128/AAC.45.5.1402-
1406.2001.

20. Ryu KH, Kim MK, Jeong YB. A recent study on the antimicrobial sensitivity of the organisms that cause urinary tract infection. Korean J Urol. 2007;48:638-45. doi: 10.4111/ kju.2007.48.6.638.

21. Amiri P, Pournajaf A, Shavalipour A, Tayebi Z, Goudarzi $\mathrm{H}$, Eslami G, et al. Evaluation of antimicrobial resistance in the Beta-lactamase Producing Escherichia Coli Isolated from Urinary Tract Infection in the Patients Referring to Taleghani Hospital of Tehran. Tabari J Prev Med. 2015;1:1119.

22. Badrhadah A, PiriI K, Mansouri K. Antiproliferative effects of some fractions of Elaeagnus angustifolia L. Flower and aerial parts of Nepeta crispa L. on K562 leukemic cells. Iran J Med Aromat Plant. 2015;31:881-890.

23. Sefidkon F, Jamzad Z, Mirza M. Chemical composition of the essential oil of five Iranian Nepeta species (N. crispa, N. mahanensis, N. ispahanica, N. eremophila and N. rivularis). Flavour Frag J. 2006; 21:764-7. doi: 10.1002/ffj.1668.

24. Sonboli A, Salehi P, Yousefzadi M. Antimicrobial activity and chemical composition of the essential oil of Nepeta crispa Willd, from Iran. Z Naturforsch C. 2004;59:653-6.

25. Mahboubi MO, Kazempour NA, Ghazian FA, Taghizadeh MO. Chemical composition, antioxidant and antimicrobial activity of Nepeta persica Boiss. essential oil. Herba Polonica. 2011;57:62-71.

26. Mozaffarian V. Identification of medicinal and aromatic plants of Iran. Tehran: Farhang Moaser; 2012. [Persian].

27. Yildirim AB, Karakas FP, Turker AU. In vitro antibacterial and antitumor activities of some medicinal plant extracts, growing in Turkey. Asian Pac J Trop Med. 2013;6:16-24. doi: 10.1016/S1995-7645(13)60106-6.

28. Thoppil RJ, Harlev E, Mandal A, Nevo E, Bishayee A. Antitumor activities of extracts from selected desert plants against HepG2 human hepatocellular carcinoma cells. Pharm Biol. 2013;51:668-74. doi: 10.3109/13880209.2012.749922.

29. Sarkhail P, Amin G, Shafiee A. Composition of the essential oil of Phlomis olivieri Benth, from north of Iran. Daru. 2006;14:71-4.

30. Turker AU, Yıldırım AB. Evaluation of antibacterial and antitumor activities of some Turkish endemic plants. Trop J Pharm Res. 2013;12:1003-10. doi: 10.4314/tjpr.v12i6.20.

31. Mojab F, Poursaeed M, Mehrgan H, Pakdaman S. Antibacterial activity of Thymus daenensis methanolic extract. Pak J Pharm Sci. 2008;21:210-3.

32. Valant-Vetschera KM, Roitman JN, Wollenweber E. Chemodiversity of exudate flavonoids in some members of the Lamiaceae. Biochem Syst Ecol. 2003;31:1279-89. doi:10.1016/s0305-1978(03)00184-4.

33. Jamzad Z, Grayer RJ, Kite GC, Simmonds MS, Ingrouille M, Jalili A. Leaf surface flavonoids in Iranian species of Nepeta (Lamiaceae) and some related genera. Biochem Syst Ecol. 2003;31:587-600. doi: 10.1016/S0305-1978(02)00221-1.

Copyright (C) 2019 The Author(s); Published by Nickan Research Institute. This is an open-access article distributed under the terms of the Creative Commons Attribution License (http://creativecommons.org/licenses/by/4.0), which permits unrestricted use, distribution, and reproduction in any medium, provided the original work is properly cited. 